\title{
Uma revisão sobre as propriedades farmacológicas, morfoanatomia e toxicicidade de Xanthium Cavanillesii Schouw (Asteraceae)
}

\author{
Daiane Flores Dalla Lana*, Raquel Medina Martins Necchi**, \\ Rosana Casoti**k, Melânia Palermo Manfron ${ }^{\star \star \star \star}$
}

Resumo: 0 gênero Xanthium, originário da América do Sul é representado por um número relativamente limitado de espécies distribuídas em vários países. Xanthium cavanillesii Schouw (Asteraceae), conhecida popularmente como carrapicho, distribui-se pela Europa e outras regiões do mundo. A espécie possui propriedades anti-inflamatória, antimicrobiana e anticarcinogênica e é usada popularmente para o tratamento da febre e gripe, além de outras doenças. É considerada uma planta infestante por se propagar e contaminar outras culturas, podendo causar intoxicações em animais quando ingerida na fase de plântula ou brotamento. Este estudo propõe-se a apresentar, comparar e discutir trabalhos que envolvam as propriedades farmacológicas, a morfoanatomia e a toxicidade de $X$. cavanillesii, através de uma revisão bibliográfica, afim de abranger todas as características relevantes da planta relacionando-as com suas ações terapêuticas e atividades biológicas.

Descritores: Xanthium cavanillesii, propriedades, morfoanatomia, toxicidade.

\section{A review about pharmacological properties, morphoanatomy and toxicity of Xanthium Cavanillesii Schouw (Asteraceae)}

\begin{abstract}
The genus Xanthium, originating in South America is represented by a relatively limited number of species distributed in several countries. Xanthium cavanillesii Schouw (Asteraceae), popularly known as burr, is distributed through Europe and other regions of the world. The specie has anti-inflammatory, antimicrobial and anticarcinogenic properties and is used popularly for the treatment of fever, flu, besides other diseases. It is considered a weed by to propagate and contaminate other cultures, could cause intoxications in animals when ingested in the seedling stage or budding. This study intends to present, compare and discuss academic works involving the pharmacological properties, the morphoanatomy and the toxicity of $X$. cavanillesii, through a literature review, in order to cover all the relevant characteristics of the plant relating them to their therapeutic and biological activities.
\end{abstract}

Descriptores: Xanthium cavanillesii, properties, morphoanatomy, toxicity.

*Graduanda em Farmácia na Universidade Federal de Santa Maria (UFSM), Santa Maria, RS, Brasil.

${ }^{* *}$ Doutoranda em Ciências Farmacêuticas na Universidade Federal de Santa Maria (UFSM), Santa Maria, RS, Brasil.

${ }^{* * *}$ Mestranda em Ciências Farmacêuticas na Universidade Federal de Santa Maria (UFSM), Santa Maria, RS, Brasil.

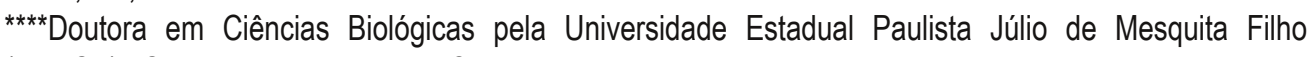
(UNESP), SP, Brasil.Doutora em Ciências Biológicas pela Universidade Estadual Paulista Júlio de Mesquita Filho (UNESP), SP, Brasil. Docente no Departamento de Farmácia Industrial na Universidade Federal de Santa Maria (UFSM), Santa Maria, RS, Brasil. 


\section{Introdução}

O trabalho da natureza e do homem vem definindo espécies de plantas com propriedades medicinais ao longo dos tempos. No reino Plantae observa-se uma grande variedade de espécies empregadas no combate às enfermidades diversas, que durante séculos foram a única fonte de agentes terapêuticos para o homem. ${ }^{1}$ Segundo Brush et al (2000), as plantas atuam como fontes de agentes terapêuticos, modelos para novos medicamentos sintéticos ou ainda como material de partida para a produção semi-sintética de moléculas de alta complexidade. Devido ao crescente uso das plantas medicinais torna-se necessário o estudo dos seus constituintes, do mecanismo de ação terapêutica e a identificação de princípios ativos responsáveis pelas diversas atividades biológicas. ${ }^{2}$

Xanthium cavanillesii Schouw (X. cavanillesii), pertencente à família Asteraceae e tribo Heliantheae, é considerada uma planta medicinal. ${ }^{3}$ Conhecida popularmente como carrapicho, carrapichão e carrapicho de carneiro, ${ }^{4}$ apresenta como sinonímia $X$. americanum Walt., X. canadense Mill., X. echinatum Murr. e X. orientale L. ${ }^{5}$

$X$. cavanillesii é originária da América do Sul, distribui-se na Europa e em outras regiões do mundo, preferindo solos úmidos e terrenos abandonados como hábitat. ${ }^{5}$ É considerada uma planta infestante, do verão anual, cuja reprodução é apenas sexuada. As mudas são produzidas no início da primavera e as plantas crescem durante toda essa estação; no verão, começam a florescer e após ocorre 0 amadurecimento dos frutos. ${ }^{6}$

A disseminação da espécie é feita pelo homem e pelos animais. Propaga-se pelas sementes através de dispersão do tipo anemocórica e conserva seu poder germinativo por muitos anos. ${ }^{7} O$ carrapicho é uma planta encontrada em potreiros, pastagens e cultivos como a soja, na qual a sua propagação é indesejada visto que suas sementes são consideras impurezas em outras culturas. ${ }^{6}$

0 presente estudo tem como objetivo realizar uma revisão bibliográfica sobre as propriedades farmacológicas, morfoanatomia e toxicidade de $X$. cavanillesii, relacionando-se as características específicas e relevantes da espécie com as suas ações terapêuticas e atividades biológicas.

\section{Metodologia}

Realizou-se um estudo de revisão bibliográfica sistemática com pesquisas em vários bancos de dados como nas coleções online de pesquisa científica da Elsevier Editora (ScienceDirect), na biblioteca virtual de saúde do National Institute of Health (PUBMED) e no Portal de periódicos da Universidade Federal de Santa Maria (UFSM), no período de outubro a novembro de 2011. O principal termo utilizado nas buscas eletrônicas foi "Xanthium cavanillesii Schouw".

A pesquisa foi realizada obtendo-se 60 publicações, as quais foram criteriosamente analisadas para integrar o presente trabalho de revisão. A seleção das produções científicas desenvolveu-se a partir da leitura completa de todos os trabalhos encontrados e

Saúde (Santa Maria), v.38, n.1, p. 57-70, 2012. Uma revisão sobre as propriedades farmacológicas, morfoanatomia e toxicicidade de xanthium cavanillesii schouw (ASTERACEAE) posteriormente priorizaram-se as obras que continham características específicas da planta em estudo. Foram excluídos os trabalhos que não estavam disponíveis na íntegra ou não possuíam referências bibliográficas adequadas. 


\section{Família Asteraceae}

A família Asteraceae, também conhecida como Compositae, é uma das maiores entre as angiospermas e está bem distribuída no Brasil. É composta por 1.535 gêneros e aproximadamente 23.000 espécies, reunidas em três subfamílias e 17 tribos. $^{8}$ É predominante em regiões do cerrado, área de elevada biodiversidade vegetal, ocorrendo ainda em menor proporção em outros ecossistemas. ${ }^{9}$ São representadas por espécies herbáceas, anuais ou perenes, subarbustivas ou arbustivas ${ }^{10}$ e caracterizam-se, geralmente, por possuírem inflorescências agregadas denominadas de capítulos, os quais atuam como uma unidade de polinização. ${ }^{11}$ Não há um modo especializado de polinização, o que facilita a conquista de novos ambientes. ${ }^{10}$

As classes de moléculas mais investigadas nessa família são os terpenóides e os compostos fenólicos que, além da diversidade estrutural, apresentam potencial farmacológico. Dentre os terpenóides, os diterpenos e as lactonas sesquiterpênicas são os mais estudados e, dentre os fenóis, destacam-se os flavonóides e, mais recentemente, os ácidos clorogênicos. ${ }^{12}$ Muitas plantas da família possuem importância econômica e biológica, sendo empregadas na terapêutica (fitoterápicos), na medicina popular e na alimentação. Como exemplos de espécies da família economicamente importantes pode-se citar a arnica (Arnica montana), a alface (Lactuca sativa), a chicórea (Cichorium intybus), a camomila (Matricaria chamomilla), o guaco (Mikania glomerata) e o falso boldo (Vernonia condansata). ${ }^{11}$

A tribo Heliantheae é a segunda maior da família Asteraceae, com mais de 2.500 espécies. Dentre as espécies mais importantes, pode-se citar o girassol (Helianthus anuus), que possui terpenóides com atividades biológicas e é empregado na produção de óleo comestível; o margaridão (Tithonia diversifolia) que é uma planta infestante cujas substâncias possuem atividade anti-inflamatória e alelopática; a equinácea (Echinacea augustifolia), cujo fitoterápico é um dos mais vendidos imunoestimulantes do mundo. ${ }^{13} \mathrm{O}$ gênero Xanthium faz parte desta tribo. ${ }^{14}$

\section{Gênero Xanthium}

O gênero Xanthium representa um número limitado de espécies, distribuídas em quase todas as partes do mundo, ${ }^{15} \mathrm{em}$ torno de 30 espécies já foram atribuídas ao gênero. ${ }^{16}$

As diferentes espécies apresentam frutos que variam muito em tamanho, cor, número e comprimento dos espinhos, porém a composição fitoquímica das plantas do gênero é muito semelhante pois estudos relatam a presença de sesquiterpenos xantanolídeos na maioria das espécies. ${ }^{17}$ Os Sesquiterpenos xantanolídeos apresentam atividade antibacteriana frente ao Staphylococcus aureus resistente à meticilina (MRSA) $)^{18}$ e atividade citotóxica para linhagens de células humanas de câncer. ${ }^{19}$

Algumas espécies de Xanthium são usadas pelas suas propriedades medicinais no tratamento de febre, leucoderma e herpes. ${ }^{3}$ Embora apresentem ações terapêuticas, Xanthium sp. são plantas invasoras de culturas anuais como soja e outras. ${ }^{16}$ Portanto, 0 gênero Xanthium apresenta múltiplas propriedades benéficas, porém a adaptação e 0 desenvolvimento de cada espécie ao meio exigem cautela e cuidados para se evitar prejuízos aos produtores. ${ }^{20}$

Saúde (Santa Maria), v.38, n.1, p. 57-70, 2012. Lana, D. F. D.; Necchi, R. M. M.; Casoti, R.; Manfron, M. P. 


\section{Xanthium cavanillesii Schouw}

Xanthium cavanillesii é uma planta lenhosa que chega a atingir 2,5 metros de altura, desenvolve um extenso sistema radicular com raízes longas e fortes. Possui forma ereta, é ramificada, com hastes cobertas por pêlos curtos e grossos. ${ }^{3}$ As folhas, verdes escuras, apresentam $10-20 \mathrm{~cm}$ de comprimento e $15 \mathrm{~cm}$ de diâmetro, são alternas, lobadas e grosseiramente dentadas sendo semelhantes a uma folha de uva mas com uma textura mais áspera e nervuras mais proeminentes; possuem a face superior verde e pubescente e a face inferior mais clara e pilosa. ${ }^{6}$ As flores discretas em forma de cachos apresentam-se no final dos muitos ramos. Cada carrapicho contém duas sementes oblongas, marrom ou pretas, de 6-10mm de comprimento. Os frutos (Figura 1) 21 $^{21}$ do tipo aquênio, são densamente cobertos de espinhos e se formam nas extremidades dos ramos da planta. ${ }^{22} O$ fruto é envolto pelo invólucro gamófilo, indeiscente, constituindo o pseudofruto. ${ }^{5}$

Os aspectos morfoanatomicos dessa espécie a caracterizam e a diferenciam. Por exemplo, as folhas de $X$. cavanillesii são mais triangulares e maiores que as demais e 0 fruto é o maior de todos, ${ }^{22}$ comparando-se com as outras espécies do gênero. Outro aspecto que a identifica em diferentes regiões é o padrão de isoenzimas peroxidase, ${ }^{23}$ essas enzimas fazem parte da genética parcial da planta, sofrendo variações de acordo com o clima, temperatura e condições de cultivo. $O$ carrapicho típico da América do Sul vai apresentar um padrão de peroxidase que o diferencia dos demais e isso é útil na separação taxonômica das espécies de Xanthium. ${ }^{24}$

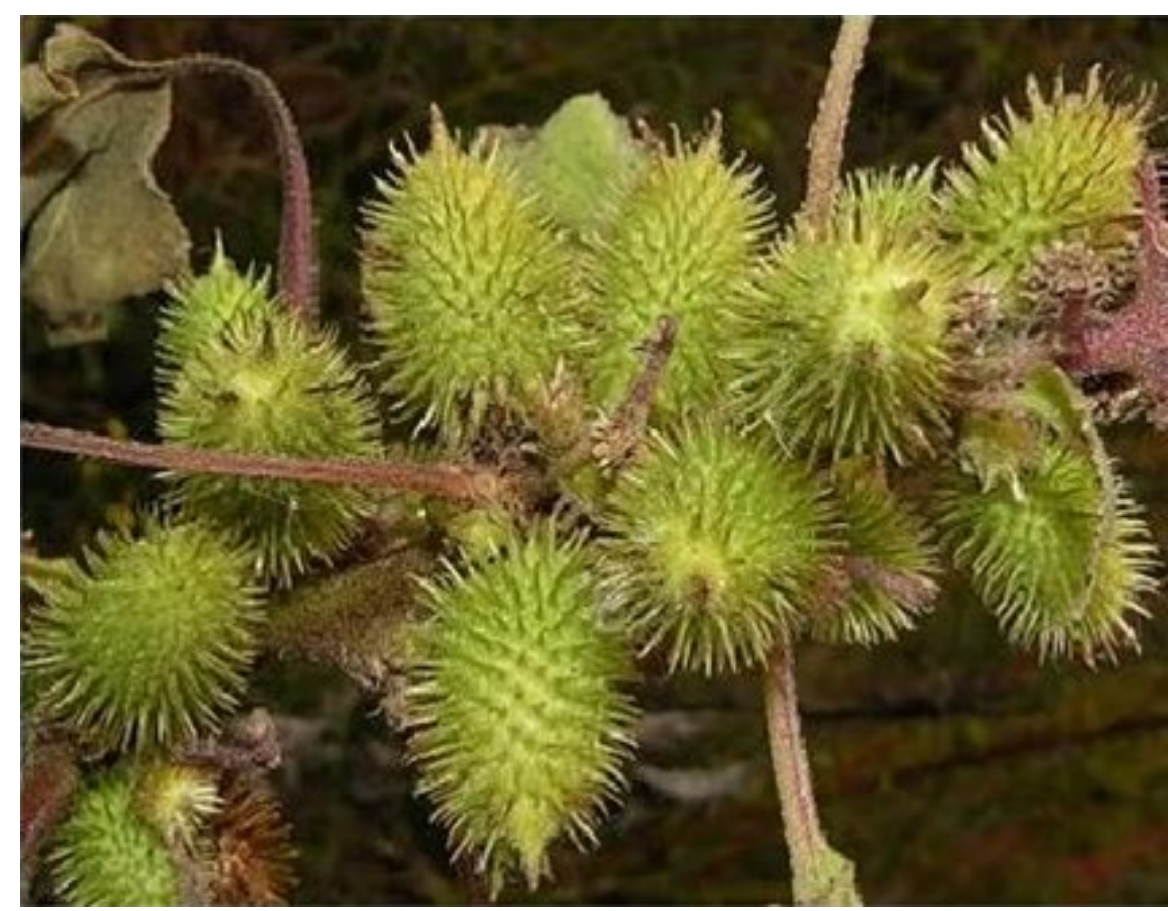

Figura 1 - Frutos de Xanthium cavanillesii Schouw (Fonte: Uruguay's wildlife and Nature sanctuaries)

Saúde (Santa Maria), v.38, n.1, p. 57-70, 2012. Uma revisão sobre as propriedades farmacológicas, morfoanatomia e toxicicidade de xanthium cavanillesii schouw (ASTERACEAE)
A classificação taxonômica é muito importante para realizar-se um reconhecimento concreto da espécie, a qual possui muitos usos populares. A saber, a infusão das folhas, sementes e raízes de $X$. cavanillesii, usada na medicina popular, possui muitas ações terapêuticas. ${ }^{5}$ As folhas são usadas pelas suas propriedades depurativas, antiespasmódica, 
antissépticas e para 0 tratamento de úlceras; ${ }^{25}$ também na cura da gripe, problemas dos pulmões, tosse e tuberculose. ${ }^{26}$ Popularmente, 0 que é mais usado é o chá das folhas como febrífugo e contra gripe. ${ }^{27}$ Este farmacógeno apresenta tão grande valor medicinal que é até mesmo indicado para tumores, gangrena e câncer. ${ }^{28}$

As sementes e raízes são diuréticas e antidisentéricas. Os frutos, na forma de cinzas, são empregados principalmente na cura de lesões da pele, por terem ação antisséptica e cicatrizante. A infusão de todas as partes da planta tem propriedades sudoríficas e antitetânicas, porém pode apresentar efeito laxativo. ${ }^{28}$

Devido ao seu amplo uso popular, investigou-se a composição química da espécie responsável pelas evidentes propriedades farmacológicas e com isso obteve-se 0 isolamento de alguns metabólitos secundários. De acordo com Cumando et al (1992) e Malik et al (1993) espécies de Xanthium produzem xantanolídeos. ${ }^{29,30}$ De Riscala et al (1994) observaram que 0 extrato clorofórmico das partes aéreas de $X$. cavanillesii contém vários xantanolídeos conhecidos, um novo tipo de xantanolídeo e um bis-norxantanolídeo. ${ }^{17}$

O componente principal dos xantanolídeos é uma lactona sesquiterpênica denominada xantumina (1b) e o componente secundário é o seu derivado diidro (2). Outras lactonas isoladas em quantidades menores foram a hidroxitomentosina (1a), o epóxido (3a), este último acompanhado de seu derivado diidro (3b) e o novo bis-norxantanolídeo (4). A estrutura química destes componentes (Figura 2) ${ }^{17}$ foi elucidada por Espectrometria de massa e Ressonância Magnética Nuclear (RMN). ${ }^{17}$

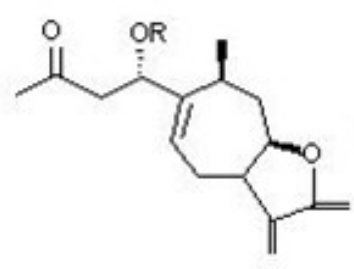

1 a $R=H$

b $R=A_{C}$

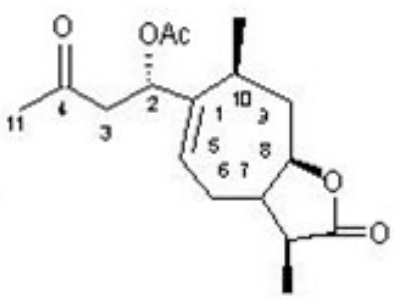

2

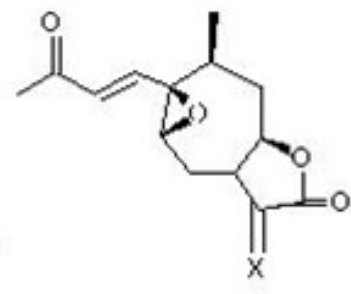

3 a $\mathrm{X}=\mathrm{CH}_{2}$ $\mathrm{b} X=\mathrm{B}-\mathrm{Me}, \mathrm{H}$

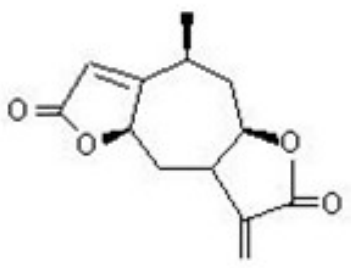

Figura 2 - Estrutura química dos compostos isolados do extrato clorofórmico das partes aéreas de Xanthium cavanillesii Schouw

(Fonte: De Riscala et al. 1994)

Para o extrato hexânico das partes aéreas de $X$. cavanillesii observou-se na cromatografia em coluna (CC) duas frações, uma com características fisico-químicas semelhantes aos triterpenos e a outra similar ao colesterol. Algumas substâncias foram

Saúde (Santa Maria), v.38, n.1, p. 57-70, 2012. Lana, D. F. D.; Necchi, R. M. M.; Casoti, R.; Manfron, M.P. ISSN 2236-5843 
isoladas nas duas frações e por RMN, espectros de massa e pontos de fusão identificou-se 0 24-metilencicloartanol, o lupeol e a $\beta$-amirina na fração semelhante aos triterpenos e 0 estigmasterol , o campesterol e o sitosterol na fração similar ao colesterol. ${ }^{31}$

No estudo de Fortuna et al (2000) observou-se para o extrato clorofórmico de $X$. cavanillesii uma intensa absorção no infravermelho $\left(1770 \mathrm{~cm}^{-1}\right)$, o que indica a presença de lactonas. ${ }^{31}$ Os compostos isolados deste extrato foram os mesmos relatados por De Riscala et al (1994): a xantumina, a hidroxitomentosina, o epóxido e o novo bis-norxantanolídeo. ${ }^{17} \mathrm{Na}$ avaliação sobre a germinação de Lactuca sativa os extratos hexânico e clorofórmico de $X$. cavanillesii demonstraram de baixa a moderada interferência na germinação da alface, não apresentando, por tanto, atividade alelopática significativa. ${ }^{31}$ As plantas maduras são rejeitadas por herbívoros, provavelmente devido ao alto teor de lactonas sesquiterpênicas, principais metabólitos secundários da espécie, que dão um sabor muito amargo à planta. ${ }^{31}$

Os metabólitos secundários são micromoléculas com diversidade e complexidade estrutural, que possuem uma distribuição geralmente restrita, ou seja, específica de um órgão da planta e possuem funções adaptativas e atividades biológicas. ${ }^{12}$ Para $X$. cavanillesii, a partir do conhecimento dos metabólitos secundários constituintes da espécie, muitas foram as atividades biológicas e farmacológicas investigadas, tais como: atividade antimicrobiana, atividade anti-inflamatória/cicatrizante e atividade antiulcerogênica.

As lactonas sesquiterpênicas presentes em $X$. cavanillesiil ${ }^{17}$ apresentaram atividade antimicrobiana, na análise pelo método de difusão em Ágar, em especial contra bactérias Gram-positivas. ${ }^{32}$

Cerdeiras et al (2007) investigaram a atividade antimicrobiana de três tipos de extrato (aquoso, etanólico e clorofórmico) das folhas, frutos e raízes de $X$. cavanillesii através do método de difusão em Ágar ${ }^{33}$ para Bacillus subtilis, Pseudomonas aeruginosa, Staphylococcus aureus, Klebsiella pneumoniae, Mycobacterium smegmatis, Candida albicans, Saccharomyces cerevisiae. A atividade de inibição desses microrganismos foi significativa para todos os extratos, mas ainda mais notável para o extrato aquoso e os valores de Concentração Inibitória Mínima (CIM) foram relativamente baixos também para todos os extratos, especialmente em relação a $M$. smegmatis e C. albicans, ou seja, mesmo em baixas concentrações os extratos são capazes de inibir os microrganismos. ${ }^{25}$

Os extratos clorofórmico e etanólico foram submetidos a testes de toxicidade aguda oral. Não houve óbitos durante o período de observação. Os animais estavam aparentemente saudáveis, não houve mudanças no comportamento normal e não foram observadas alterações patológicas na necropsia, desta forma, apresentando baixa toxicidade oral em ratos..$^{33} \mathrm{~A}$ propriedade antimicrobiana justifica 0 uso tradicional das infusões de $X$. cavanillesii para 0 tratamento de infecções da pele e torna essa espécie uma fonte interessante de compostos antimicrobianos. ${ }^{34}$

Schmidt et al (2009) desenvolveram uma pesquisa com os extratos hexânico e etanólico de doze plantas, do sul do Brasil, utilizadas na medicina tradicional para a cicatrização de feridas. Dentre essas doze destaca-se $X$. Cavanillesii. Para comprovar o seu uso tradicional os extratos foram investigados em diversos ensaios biológicos sendo relacionados a diferentes etapas dos processos de cicatrização ${ }^{35}$.

Os extratos hexânico e etanólico de $X$. Cavanillesii inibiram completamente ou quase completamente os mediadores dos processos inflamatórios como NF-KB/DNA vinculativo, MAPK p38, TNF-a, bem como reduziram a atividade descontrolada da elastase 
serinoprotease.$^{35}$ Essas ações provocam uma perfeita cicatrização das lesões de pele e 0 alto potencial inibitório dos extratos frente às respostas inflamatórias confirma a significativa atividade anti-inflamatória de $X$. cavanillesii ${ }^{36}$

No estudo de Favier et al (2005) analisou-se o efeito preventivo dos xantanolídeos naturais, bem como uma série de derivados sintéticos sobre a formação de úlcera, induzida por etanol absoluto, em ratos. Entre os compostos testados, xantatina deu a mais forte atividade protetora. ${ }^{15} \mathrm{~A}$ ação inibitória exercida por esta molécula sobre as lesões gástricas foi altamente significativa, reduzindo a ulceração na faixa de 58-96\%, esse valor percentual de inibição da úlcera é comparável ao descrito na literatura para dehidroleucodine (DHL), a mais ativa lactona sesquiterpênica que possui este tipo de bioatividade. ${ }_{37}$ Essa ação antiulcerogênica das lactonas sesquiterpênicas é atribuída a presença de um receptor eletrofílico livre na estrutura molecular, o qual interage com compostos sulfidrilicos da mucosa gástrica, a saber, glutationa reduzida (GSH) que é um dos principais componentes antioxidantes não enzimáticos celulares. ${ }^{38}$

Penissi et al (2009), também avaliaram o efeito antiulcerogênico da xantatina, observado pela degranulação dos mastócitos induzida pelo composto sintético 48/80. Os mastócitos são componentes protetores importantes do trato gastrointestinal, com isso a sua degranulação provoca processos inflamatórios e consequentemente lesões na mucosa gástrica. 0 composto sintético 48/80 induz a degranulação dos mastócitos. Nesse estudo a xantatina inibiu consideravelmente esse composto, provando ter uma ação antiúlcera realmente eficaz. 39

Os princípios ativos das plantas, dependendo do tipo e da concentração, podem proporcionar benefícios como é o caso das propriedades farmacológicas e atividades biológicas já mencionadas para $X$. cavanillesii, mas também podem ocasionar alguns efeitos indesejáveis por apresentarem certo grau de toxicidade.

Os metabólitos secundários ativos responsáveis pela toxicidade de algumas plantas já foram identificados. ${ }^{40}$ Das plantas que causam necrose hepática aguda destaca-se $X$. Cavanillesi por conter os carboxiatractilosídeos (CAT). ${ }^{4}$ Os CAT são glicosídeos triterpenóides, isolados dos frutos e da brotação, ${ }^{41}$ responsáveis pelo quadro de insuficiência hepática aguda em ruminantes. ${ }^{4}$ Causam inibição da respiração das mitocôndrias e da síntese de ATP, inibindo o transporte de ADPIATP através da membrana da mitocôndria e alterando 0 processo de fosforilação oxidativa pelo bloqueio da translocação da adenina nucleotídeo nessa organela. Sob condições naturais, Xanthium sp. causa intoxicação aguda. ${ }^{40}$

A intoxicação pela espécie não provoca alterações morfológicas no SNC. Nos casos de insuficiência hepática, às vezes, há sinais clínicos de doença nervosa, mas não há alterações morfológicas no SNC. Sugere-se que talvez outros mecanismos, como a hipoglicemia que ocorre nessas intoxicações, possam ser responsáveis pelos sinais clínicos. ${ }^{42}$

Rissi et al (2007) constataram que a frequência de intoxicação por $X$. cavanillesii no Rio Grande do Sul, em 2007, foi de 3,07\% (em um total de 461 casos de intoxicação por plantas tóxicas). Os principais sinais clínicos observados foram anorexia, desidratação, tenesmo, incoordenação, agressividade, decúbito e cegueira. Os achados de necropsia revelam hemorragias na serosa de vários órgãos, edema das pregas do abomaso e da parede da vesícula biliar e fezes ressequidas; fígado com acentuação do padrão lobular e hidropericárdio, necrose hepatocelular centrolobular ou massiva com degeneração gordurosa de hepatócitos. ${ }^{43}$

Saúde (Santa Maria), v.38, n.1, p. 57-70, 2012. Lana, D. F. D.; Necchi, R. M. M.; Casoti, R.; Manfron, 
São poucos os dados sobre a intoxicação pelos frutos do carrapicho em bovinos, por isso Colodel et al (2000) realizaram um estudo de intoxicação experimental pelos frutos de $X$. Cavanillesii em bovinos. Estabeleceram a dose tóxica, determinaram o quadro clínicopatológico e as alterações bioquímicas associadas. Os frutos moídos foram administrados por via oral, em doses únicas ou repetidas, com intervalo semanal, a onze bovinos, dos quais, quatro morreram. Doses únicas a partir de $5 \mathrm{~g} / \mathrm{kg}$ foram letais para bovinos, sendo essa a dose letal mínima dos frutos de $X$. cavanillesii estabelecida neste experimento, porém em outros dados da literatura consta que a dose letal mínima seria de $3 \mathrm{~g} / \mathrm{kg}$. A dose de 3 $\mathrm{g} / \mathrm{kg}$ apenas produziu sinais clínicos no animal que se recuperou. Dos três bovinos que receberam $3 \mathrm{~g} / \mathrm{kg}$, dois não adoeceram e o outro se recuperou bem após apresentar sinais clínicos e alterações patológicas semelhantes às observadas em bovinos que morreram intoxicados por $X$. Cavanillesii. ${ }^{44}$

Contatou-se hipoglicemia e elevação dos níveis séricos de aspartato aminotransferase (AST) nos bovinos que apresentaram sinais clínicos da intoxicação, representando a presença de um dano hepático. Os sinais clínicos observados nos bovinos foram compatíveis com aqueles previamente publicados e já relatados sobre bovinos intoxicados natural ou experimentalmente por Xanthium sp.: desidratação, tenesmo, incoordenação, dentre outros. ${ }^{44}$

Loretti et al (1999) propuseram um estudo de intoxicação experimental pelos frutos de $X$. cavanillesii em ovinos. Os frutos moídos foram administrados por via oral, em doses únicas ou fracionadas, a 15 ovinos adultos, dos quais nove morreram. Doses a partir de $2 \mathrm{~g} / \mathrm{kg} \mathrm{em}$ uma única administração foram letais. Doses únicas de $1,25 \mathrm{~g} / \mathrm{kg}$ e doses de $2,5 \mathrm{~g} / \mathrm{kg}$ subdivididas em duas administrações diárias (1,25 g/kg em dois dias consecutivos) não causaram a intoxicação. Sinais clínicos foram observados apenas nos animais que morreram e geralmente iniciavam entre 5 horas e 20 horas após o início da administração dos frutos. ${ }^{46}$ A evolução do quadro clínico foi superaguda ( 90 minutos a 3 horas) ou aguda (9 a 13 horas). Sinais clínicos incluíam apatia, anorexia, hipomotilidade ruminal, tremores musculares generalizados, incoordenação motora, andar rígido, relutância em caminhar, instabilidade, quedas e decúbito. ${ }^{44}$

A principal lesão microscópica nos ovinos foi observada no fígado, ocorreu nos nove ovinos necropsiados e caracterizava-se por acentuação do padrão lobular na superfície capsular e de corte e a mais importante alteração histopatológica, encontrada em todos os ovinos necropsiados, consistia em acentuada necrose coagulativa hepatocelular centrolobular ou massiva. ${ }^{46}$ Observa-se, então, que os sinais clínicos, os dados de necropsia e as alterações microscópicas foram semelhantes aos descritos na intoxicação espontânea e experimental por Xanthium sp. em bovinos.

A intoxicação espontânea por Xanthium sp. tem sido descrita em suínos ${ }^{47}$, bovinos ${ }^{48}$, ovinos $^{46}$ e equinos ${ }^{49}$. Driemeier et al (1999) descreveram a ocorrência de dois surtos de intoxicação espontânea de bovinos pelos frutos de $X$. cavanillesii em dois estabelecimentos dos municípios de Casca e Cachoeira do Sul, no Rio Grande do Sul, no final de abril e meados de agosto de 1996, cujos sinais clínicos foram muito semelhantes aos já mencionados para os casos de intoxicação experimental pelo carrapicho. ${ }^{50}$

A toxicidade é atribuída à presença de saponinas e a presença dos glicosídeos CAT, já

Saúde (Santa Maria), v.38, n.1, p. 57-70, 2012. Uma revisão sobre as propriedades farmacológicas, morfoanatomia e toxicicidade de xanthium cavanillesii schouw (ASTERACEAE) relatados também nos cotilédones de sementes e plântulas de outras espécies do gênero. ${ }^{51}$

As plantas hepatotóxicas da Região Sul que, em condições naturais, causam em bovinos um quadro clínico-patológico semelhante ao observado na intoxicação por $X$. cavanillesii pertencem ao gênero Cestrum e incluem C. intermedium (Santa Catarina e Rio 
Grande do Sul), C. corymbosum var. hirsutum e C. parqui. Nesta região do país, a distribuição, os diferentes habitats e a ocorrência sazonal destas plantas permitem que, na maior parte dos casos, o diagnóstico diferencial da intoxicação por $X$. cavanillesii seja feito sem dificuldades. ${ }^{50}$

Como X. cavanillesii é considerada uma planta infestante nociva em áreas de produção agrícola e agropecuária, a sua introdução deve ser evitada. ${ }^{52} \mathrm{O}$ método mais provável de introdução da espécie em determinada área é pelo fruto que fica preso em roupas e pêlos. Dessa forma, pode-se ajudar na prevenção verificando esses ítens e até mesmo usando-se animais de controle, em especial em áreas sabidamente infestadas com carrapichos. Medidas profiláticas variam de planta a planta, dependendo da espécie, do habitat e das condições nas quais ocorrem as intoxicações. Algumas medidas importantes incluem erradicar as plantas tóxicas das pastagens, não permitir 0 acesso dos animais em áreas invadidas na qual a erradicação não foi possível e tomar muito cuidado na transferência de animais de um pasto a outro. ${ }^{52}$

As infestações pequenas podem ser removidas manualmente antes da semeadura, certificando-se de retirar toda a raiz. Se a semente madura está presente, ela deve ser coletada e queimada para se evitar disseminação. Infestações maiores devem ser pulverizadas com um herbicida registrado antes que a semente amadureça. ${ }^{53}$

A fim de se verificar possibilidades que interrompam o desenvolvimento do carrapicho, 0 estudo de Logarzo et al (2002) propõe um controle biológico. A busca de inimigos naturais para a espécie, na América do sul, foi realizada na Argentina e um candidato que surgiu a partir da seleção foi 0 besouro Apagomerella versicolor (Boheman) (Coleoptera: Cerambycidae). $O$ ataque de $A$. versicolor, na fase de larva e adulto, reduziu a produção de frutos em $66 \%$ e matou as plantas jovens, provando ter atributos de um agente eficaz. Esses atributos, juntamente com a tolerância ao frio, a tolerância de imersão em água e a ampla distribuição de habitat da floresta tropical ao deserto, sugerem que o besouro seria uma forma válida como controle biológico. ${ }^{6}$

Além desse agente, destaca-se o uso particularmente promissor do fungo Puccinia xanthii Schw., o qual causa o aparecimento rápido de necrose foliar nas plantas atingidas por produzir metabólitos tóxicos as espécies de Xanthium. ${ }^{54}$

\section{Conclusão}

Este trabalho auxilia na descoberta de todos os aspectos importantes já relatados para Xanthium cavanillesii, tais como suas propriedades farmacológicas, morfoanatomia e toxicidade. $X$. cavanillesii é uma planta lenhosa diferenciada das demais espécies do gênero por suas características morfoanatômicas como folhas triangulares e grandes. Na medicina popular seu principal uso é como febrífugo e para o tratamento da gripe.

$\mathrm{Na}$ composição química da espécie relata-se a presença significativa dos xantanolídeos, que são lactonas sesquiterpênicas responsáveis por algumas atividades biológicas, e outros metabólitos secundários minoritários. Diferentes extratos de $X$. cavanillesii apresentaram atividade antimicrobiana, atividade anti-inflamatória/cicatrizante e atividade antiulcerogênica, 0 que justifica os usos populares da planta em infusos e decoctos.

Saúde (Santa Maria), v.38, n.1, p. 57-70, 2012. Lana, D. F. D.; Necchi, R. M. M.; Casoti, R.; Manfron, M. P. ISSN 2236-5843 
A espécie por apresentar em sua constituição fitoquímica os carboxiatractilosídeos, pode causar quadros de intoxicação grave em suínos, bovinos, ovinos e equinos, quando ingerida na fase de plântula ou brotamento. Além disso, pode contaminar cultivos agrícolas, sendo considerada uma planta infestante nociva. Para prevenção e controle de $X$. cavanillesii, recomenda-se erradicar o carrapicho das pastagens, não permitindo 0 acesso dos animais em áreas invadidas.

\section{Referências}

1. Vieira PC. Estratégias para o isolamento de princípios ativos de plantas. In: VII Jornada Paulista de Plantas Medicinais; 2005 fev. 15-17; São Paulo. São Paulo: ADEn-Seção-SP; 2005. p.20-21.

2. Bruschi ML, Franco SL, Novello $C R$. Projeto de manipulação e desenvolvimento de medicamentos e correlatos a base de plantas na disciplina de farmacotécnica. In: Anais do XVI Simpósio de Plantas Medicinais do Brasil; 2000 set 20-23; Recife (UFPE). Recife: Congrade- Seção- PE; 2000. p. 248-249.

3. Hsu FL, Chen Y, Cheng J. Caffeic acid as active principle of fruits Xanthium strumarium to lower plasma glucose in diabetics rats. Rev Planta Med. 2000; 66(1): 228-230.

4. Santos JCA, Riet-Correa F, Simões SVD, Barros CSL. Patogênese, sinais clínicos e patologia das doenças causadas por plantas hepatotóxicas em ruminantes e eqüinos no Brasil. Pesq Vet Bras. 2008; 28 (1): 1-14.

5. Groth D. Identificação botânica de plantas e sementes de espécies invasoras na cultura de soja. Rev Bras Sementes. 1980; 02(3): 59-95.

6. Logarzo G, Gandolfo D, Cordo H. Biology of Apagomerella versicolor (Boheman) (Coleoptera: Cerambycidae) in Argentina, a candidate for biological control of cocklebur (Xanthium sp.). Biological Control. 2002; 25 (02): 22-29.

7. Venzke TS, Seibel GP, Costa MAD. Características da síndrome de dispersão em uma comunidade vegetal ocorrente em um terreno baldio em Pelotas, RS. In: XVII Congresso de Iniciação Científica, X Encontro de Pós Graduação, 2008 Jan.15-18; Rio Grande do Sul. Rio Grande do Sul: Pelotas- Seção- RS; 2008. p. 30-31.

8. Bremer K. Asteraceae: Cladistics and classification. Portland- Timber Press, 1994.

9. Gottliebe OR, Kaplan MAC, Borin MRMB. Biodiversidade: um enfoque químico - biológico. $1^{\text {aa }}$ Ed. Rio de Janeiro: Ed. UFRJ, 1996.

10. Barroso GM, Peixoto AL, Costa GC, Ichaso CLF, Guimarãe EF, Lima HC. Sistemática de angiospermas do Brasil. $2^{\mathrm{a}}$ Ed. Alagoas: Ed. Universidade Federal de Viçosa, 1991.

11. Weberling, F. Morphology of flowers and inflorescenses. $1^{\mathrm{a}}$ Ed. Cambrigde: Ed. Cambrigde University Press, $1989.405 \mathrm{p}$.

12. Gobbo-Neto L, Lopes NP. Identification of chlorogenic acids, sesquiterpene lactones, and flavonoids in the Brazilian arnica Lychnophora ericoides mart. (Asteraceae) leaves by HPLC-DAD-MS and HPLC-DAD-MS/MS and a validated HPLC-dad method for their simultaneous analysis. J agric Food chem. 2008; 56(1): p. 1193 1204.

13. Guimarães D.O. Prospecção química e biológica em fungos endofiticos associados à Viguiera arenaria (Asteraceae): [s.n.], 2006. 208 p. Dissertação (Mestrado) - Faculdade de Ciências Farmacêuticas de Ribeirão

Saúde (Santa Maria), v.38, n.1, p. 57-70, 2012. Uma revisão sobre as propriedades farmacológicas, morfoanatomia e toxicicidade de xanthium cavanillesii schouw (ASTERACEAE)
Preto, Universidade de São Paulo, Ribeirão Preto, 2006.

14. Love D, Dansereau P. Biosystematic studies on Xanthium: taxonomic appraisal and ecological status. J Bot. 1959; 37(2): 173-208. 
15. Favier LS, Maria AOO, Wendel GH, Borkowski EJ, Giordano OS, Pelzer L et al. Anti-ulcerogenic activity of xanthanolide sesquiterpenes from Xanthium cavanillesii in rats. J ethnopharmacol. 2005; 100(3): 260-267.

16. Holm LG, Plucknett DL, Pancho JV, Herberger JP. The world's worst weeds. East-West Center, University Press of Hawaii, Honolulu. 1977; 26(1): 73-92.

17. De Riscala EC, Fortuna MA, Catalán CAN, Díaz JG, Herz W. Xanthanolídeos e um bis-norxanthanolídeo de Xanthium cavanillesii. Phytochemistry. 1994; 35(6): 1588- 1589.

18. Sato Y, Oketani H, Yamada T, Singyouchi K, Ohtsubo T, Kihara M et al. The xanthanolide with potent antibacterial activity against methicillin-resistant Staphylococcus aureus. J Pharm Pharmacol. 1997; 49(3): 1042-1044.

19. Kinghorn AD, Farnsworth NR, Soejarto DD, Cordell GA, Pezutto JM, Udeani GO et al. Novel strategies for the discovery of plant-derived anti-cancer agents. Rev Pure Appl Chem. 1999; 71(5): 1611-1618.

20. Kissmann K, Groth D. Plantas Infestantes e Nocivas. 2ª ed. São Paulo: Ed. BASF; 1992.

21. Uruguay's wildlife and Nature sanctuaries. 2010. Available from: http://uruguay1.blogspot.com.

22. Martin RJ. Distribution, ecology and control of Xanthium species. PhD Thesis. Australian: [s.n.], 1981.126p. Thesis (PhD) - Australian National University, 1981.

23. Kobrehel K, Gautier MF. Variability in peroxidase isozymes in wheat and related species. Can J Bot. 1974; 52(1): 754-755.

24. McMillan C. The Xanthium strumarium complexes in Australia. Aus J Bot. 1975; 23(3): 173-192.

25. Cerdeiras MP, Alborés S, Etcheverry S, Lucián V, Soubes M, Vázquez A. antimicrobial activity of Xanthium cavanillesii extracts. Biol pharm. 2007; 45(3): 251-254.

26. Vendruscolo GS, Simões CMO, Mentz LA. Etnobotânica no Rio Grande do Sul: Análise comparativa entre o conhecimento original e atual sobre as plantas medicinais nativas. In: I Exposição do instituto Anchietano de Pesquisas; 2005 out. 28-30; São Leopoldo. São Leopoldo: Seção- n 56- RS; 2005. p.285-322.

27. Davis W. The development of dormancy in seeds of cocklebur (Xanthium). Am J Bot. 1930; 17(2):77-87.

28. Marzocca A (1993). Manual de malezas. $4^{a}$ ed. Argentina: Ed. Hemisferio Sul S.A; 1993. p. 504-506.

29. Cumando J, Marinoni G, De Bernardi M, Vidari G, Finzi PP. New sesquiterpenes from Xanthium catharticum. J Nat Prod. 1992; 54(3): 457- 460.

30. Malik MS, Sangwan NK, Singh K. Biological activities. Phytochemistry. 1993; 32(1): 204-206.

31. Fortuna MA, Trimarco JT, Riscalay EC, Catalán CAN. Triterpenos, Esteróles y Xanthanolides de Xanthium cavanillesii. In: IX Congresso Nacional de Recursos Naturales Aromáticos y Medicinales; 2000 jun. 17-19; Argentina. Argentina: ABEn-Seção-n015; 2000. p. 27-34.

32. Tsankova ET, Trendafilova AB, Kujumgiev Al, Galabov AS, Robeva PR. Xanthanolides of Xanthium italicum Moretti and their biological activity. Z Naturforschung C. 1994; 49(1): 154-159.

33. Barry AL, Thomsberry JC. Manual of Clinical Microbiology. Americ Soc of Microb. 1985; 30(5):23-25.

34. Lombardo A. Flora Montevidensis. $1^{0}$ ed. Montevideo: Ed. IMM; 1983.

35. Schmidt C, Fronza M, Goettert M, Geller F, Luik S, Flores EMM et al. Biological studies on Brazilian plants used in wound healing. J Ethnopharmacol. 2009; 12(2): 523-532.

36. Siedle B, Hrenn A, Merfort I. Natural compounds as inhibitors of human neutrophil elastase. Rev Bras Plantas Med. 2007; 7(3): 401-420.

37. Giordano OS, Pestchanker MJ, Guerreiro E, Saad JR, Enriz RD, Rodríguez AM et al. Structure-activity relationship in the gastric cytoprotective effect of several sesquiterpene lactones. J of Med Chem. 1992; 35(2): 2452-2458.

Saúde (Santa Maria), v.38, n.1, p. 57-70, 2012. Lana, D. F. D.; Necchi, R. M. M.; Casoti, R.; Manfron, M.P.

ISSN 2236-5843 
38. María AOM, Franchi AM, Wendel GH, Gimeno M, Guzmán JA, Giordano OS et al. Gastric cytoprotective activity of dehydroleucodine in rats. Biol and Pharm Bulletin. 1998; 21(6): 335-338.

39. Penissi AB, Vera MB, Mariani ML, Rudolph MI, Ceñal JP, Rosas JC et al. Novel anti-ulcer a, $\beta$-unsaturated lactones inhibit compound 48/80-induced mast cell degranulation. Eur J Pharmacol. 2009; 612(2): 122-130.

40. Zanoli JCC, Garcia AF, Mingatto FE. Plantas hepatotóxicas de interesse na pecuária brasileira. In: V Simpósio de Ciências (VI Encontro de Zootecnia); 2009 set. 22-24; Dracena. Dracena: BRn-Seção-SP; 2009. p. 100-104.

41. Stuart BP, Cole RJ, Gosser HS. Cocklebur (Xanthium strumarium var. strumarium) intoxication in swine: review and redefinition of the toxic principle. Vet Pathol. 1981; 18 (2): 368-383.

42. Sanches AWD, Langohr IM, Stigger AL, Barros CSL. Doenças do sistema nervoso central em bovinos no Sul do Brasil. Pesq Vet Bras. 2000; 20 (3): 113-118.

43. Rissi DR, Rech RR, Pierezan F, Gabriel AL, Trost ME, Brum JS et al. Intoxicações por plantas e micotoxinas associadas a plantas em bovinos no Rio Grande do Sul: 461 casos. Pesq Vet Bras. 2007; 27(7): 261-268.

44. Colodel EM, Driemeier D, Pilati C. Intoxicação experimental pelos frutos de Xanthium cavanillesii (Asteraceae) em bovinos. Pesq Vet Bras. 2000; 20(1):31-38.

45. Kingsbury JM. Poisonous Plants of the United States and Canada. $3^{\mathrm{a}}$ ed. New Jersey: Ed. Prentice-Hall; 1964.

46. Loretti AP, Bezerra PS, llha MRS, Barros SS, Barros CSL. Intoxicação experimental pelos frutos de Xanthium cavanillesii (Asteraceae) em ovinos. Pesq Vet Bras. 1999; 19 (2): 68-75.

47. Gava A, Barros CSL, Pilati C, Barros SS, Mori AM. Intoxicação por Ateleia glazioviana (Leg. Papilionoideae) em bovinos. Pesq Vet Bras. 2001; 21 (2): 49-59.

48. Méndez MC, Santos RC, Riet-Correa F. Intoxicação por Xanthium sp. (carrapicho) em bovinos. Boletim do Laboratório Regional de Diagnóstico (Doc 14). Editora e Gráfica Universitária, Pelotas. 1994. p. 27-30.

49. Marsh CD, Glenwood CR, Clawson AB. Cockleburs (species of Xanthium) as poisonous plants. US Dept Agric Tech Bull. 1924; $127(4): 1-24$.

50. Driemeier D, Irigoyen LF, Loretti AP, Colodel EM, Barros CSL. Intoxicação espontânea pelos frutos de Xanthium cavanillesii (Asteraceae) em bovinos no Rio Grande do Sul. Pesq Vet Bras. 1999; 19(1): $12-18$.

51. Cole RJ, Stuart BP, Lansden JA, Cox RX. Isolation, and redefinition of the toxic agent from cocklebur Xanthium strumarium. J Agric Food Chem. 1980; 28(2):13-30.

52. Martin RJ. Distribuition, ecology and control of Xanthium species. Canberra: [s.n.], 1981. 10 p. Thesis (Ph.D) - Australian National University, 1981.

53. Parsons WT, Cuthbertson EG. Noxious Weeds of Australia. $1^{\text {a }}$ ed. Australia: Inkata Press; 1992.

54. Vurro M, Evidente A, Andolfi A, Zonno MC, Giordano F, Motta A. Brefeldin A and a,b-dehydrocurvularin, two phytotoxins from Alternaria zinniae, a biocontrol agent of Xanthium occidentale. Plant Science. 1998; 138(2): 67-79.

Saúde (Santa Maria), v.38, n.1, p. 57-70, 2012. 


\section{Daiane Flores Dalla Lana}

Endereço para correspondência - Universidade Federal de Santa Maria, Av. Roraima $n^{0} 1000$ prédio 26, sala 1134. Camobi, 97105-900 - Santa Maria, RS - Brasil.

E-mail: dayalana@hotmail.com

Currículo Lattes: http://lattes.cnpq.br/2477648307638964

Recebido em 13 de março de 2012.

Aprovado em 04 de outubro de 2012. 
Saúde (Santa Maria), v.38, n.1, p. 57-70, 2012. Uma revisão sobre as propriedades farmacológicas, morfoanatomia e toxicicidade de xanthium cavanillesii schouw (ASTERACEAE) 